Japan. J. Math.

Vol. 13, No. 2, 1987

\title{
1-Generic degrees and minimal degrees in higher recursion theory ${ }^{1}$
}

\author{
By C.T. Chong
}

(Received May 7, 1985)

A project that has been carried out with much success in the study of admissible recursion theory is the investigation of the interplay between settheoretic notions such as (definable) cofinality and projectum of an admissible ordinal on the one hand, and the recursion-theoretic properties of the ordinal, particularly those relating to various notions of reducibility, on the other hand. This study has largely clarified the role of non- $\Sigma_{2}$-admissibility in recursion theory on the ordinals (cf. Chong [1] for a survey of recent results).

The methods which have been used for this study are mostly associated with the technique of priority (one notable exception is Friedman [6]). By contrast, the method of forcing, whose origin is of course set-theoretic, is not featured as prominently in these investigations. In [7], Jockusch introduced the class of $n$-generic sets (which are sets of natural numbers Cohengeneric with respect to arithmetical formulas with $n-1$ alternations of quantifiers), and studied its Turing degree-theoretic significance. As it turns out, Friedberg [5] had essentially proved his completeness theorem on Turing jumps by constructing 1-generic sets. This was observed by Simpson [10] in his generalization of the completeness theorem to all admissible ordinals. Subsequent developments showed that the notion of a 1-generic set is very useful for recursion-theoretic purposes (cf. Jockusch and Posner [8], Posner and Robinson [9]).

The degree-theoretic ordering relation between an $n$-generic set and a set of minimal degree is of particular methodological interest. This is prompted by the fact that at any stage in the construction of an $n$-generic set, all the strings (i.e. functions from a finite ordinal into 2) which satisfy the given $\sum_{n}$ relation under consideration are candidates for an initial

${ }^{1}$ Part of the work was done while the author visited Japan in summer 1984 supported by the Japanese Society for the Promotion of Science under the JSPS-NUS Exchange Programme. The author is grateful to Professor Nagayoshi Iwahori, his host scientist, and the University of Tokyo, the host institution, for the gracious hospitality. $\mathrm{He}$ also wishes to thank Professors Tosiyuki Tugue, Kanji Namba, and Nobuyoshi Motohashi for making his visit to Japan a memorable experience. 
segment of the $n$-generic set to be constructed, whereas in the construction of a set of minimal degree, only those strings lying on a given perfect closed set are eligible for consideration (thus contrasting Cohen forcing with Sacks forcing). This severe restriction makes it impossible to have an $n$-generic set of minimal degree (as can be easily shown), and hence no $n$-generic set is bounded by (i.e. recursive in) a set of minimal Turing degree. This result generalizes readily to all admissible ordinals. The converse problem, that of the existence of a set of minimal degree bounded by an $n$-generic set, turns out to be a difficult problem even in the setting of classical recursion theory. Jockusch [7] showed that no 2-generic set (hence no $n$-generic set for $n \geqq 2$ ) bounds a set of minimal degree, and this was later improved by Chong and Jockusch [4] to include those 1-generic sets which are recursive in $\emptyset^{\prime}$. Chong [3] has recently shown that this is the best possible, in the sense that there exists a 1-generic set recursive in $\emptyset^{\prime \prime}$ which bounds a set of minimal degree. Thus the problem of the ordering relation between degrees of 1-generic sets and minimal degrees has no simple answer.

In this paper we prove the result of [4], that no 1-generic set recursive in $\emptyset^{\prime}$ bounds a set of minimal degree, for all admissible ordinals. The proof of the result is not a straightforward adaptation of [4] because the method of [4], which uses the idea of constructing trees of strings of 'height $s$ ' for each finite ordinal $s$, works only for a rather limited class of admissible ordinalsthose which have a $\Sigma_{2}$-definable projection onto $\omega$. A different approach is required for the solution. We introduce instead the notion of strings having 's splittings' for each ordinal $s$ below an admissible ordinal $\alpha$, and use it to derive the desired result by constructing a 1-generic set.

This paper is largely self-contained. No knowledge of the proof in [4] is required, although an understanding of the basics of $\alpha$-recursion theory, as presented in [2], will be assumed. We fix an admissible ordinal throughout the discussion. For convenience, we will drop the prefix $\alpha$ on terms such as weakly $\alpha$-recursive, $\alpha$-recursive, $\alpha$-degree, $\alpha$-regular, $\alpha$-hyperregular etc, unless there is a possibility of confusion. We will however retain the $\alpha$ on $\alpha$-finite. The notation $A \leqq{ }_{\alpha} B$ will continue to mean ' $A(\alpha-)$ recursive in $B^{\prime}$.

Recall that a set $A \subset \alpha$ is regular if $A \cap s$ is $\alpha$-finite (i.e. an element of $L_{\alpha}$ ) for every $s<\alpha$. A is hyperregular if for all functions $f$ weakly recursive in $A, f^{\prime \prime \prime} s$ is bounded below $\alpha$ for all $s<\alpha$. As always $\alpha^{*}$ denotes the $\Sigma_{1}$ projectum of $\alpha$. This is the least ordinal $s \leqq \alpha$ for which there is a $\Sigma_{1}$ definable subset of $s$ not $\alpha$-finite. It is also known to be the least ordinal $s$ for which there exists a $\Sigma_{1}$ definable (i.e. recursive) injection mapping $\alpha$ into $s$.

We will not distinguish between a set $A$ with its characteristic function, which is a $\{0,1\}$-valued function taking the value 1 precisely at those 
ordinals which are members of $A$.

A string $p$ is an $\alpha$-finite function which is the characteristic function of an $\alpha$-finite set. The length of $p$, denoted $1 \operatorname{th}(p)$, is the least ordinal $s$ not in the domain of $p$. A string $q$ extends a string $p$ if in the domain of $p$, the two functions agree. This implies that the domain of $q$ is at least as large as the domain of $p$ (observe that the domains are ordinals less than $\alpha$ ). We write $q \geqq p$ in this case. The notation $q>p$ ( $q$ properly extends $p$ ) has its obvious meaning. Two strings such that neither extends the other are said to be incompatible. Otherwise they are compatible. The notation $p<A$ means that $p$ is extended by the characteristic function of $A$. We say in this case that $p$ is an initial segment of $A$.

Definition $A \subset \alpha$ is 1-generic if for all $\alpha$-finite collection $K$ of recursively enumerable sets of strings $R_{e}$, there is a $p<A$ such that either for each $R_{e}$ in $K, p$ extends some string in $R_{e}$, or there is an $R_{e}$ in $K$ such that no string extending $p$ lies in $R_{e}$. (Of course, $K$ means a set of indices of r.e. sets.)

Observe that the definition given here is apparently stronger than that given in Chapter 2, page 39 of [2]. As it turns out, the two definitions are equivalent. This will be shown in the appendix. We state the next result without proof ([2, Lemma 2.5]):

Proposition. If $G$ is 1-generic, then $G$ is regular and hyperregular.

Note that a 1-generic set is by necessity not recursive. A degree is 1generic if it contains a 1-generic set. A non-recursive degree is minimal if there is no non-recursive degree lying strictly below it. Whereas 1-generic degrees are easily constructed for every admissible ordinal, minimal degrees are not. In fact the problem of the existence of a minimal degree for every admissible ordinal remains to be one of the most outstanding open problems in recursion theory. Inspite of this, we may still derive the corollary below:

Theorem. Let $G$ be a 1-generic set recursive in $\emptyset^{\prime}$, and let $B$ be a nonrecursive set recursive in $G$. Then there is a 1-generic set recursive in $B$.

Corollary. No 1-generic degree recursive in $O^{\prime}$ bounds a minimal degree.

LEMMA 1. Let $A$ be a regular set recursive in $\emptyset^{\prime}$. Then there exists a recursive collection of strings $\left\{p_{v}\right\}_{v<\alpha}$ such that for all $t<\alpha, A\left|t=p_{v}\right| t$ for all sufficiently large $v$, i.e. $\lim _{v \rightarrow \alpha} p_{v}=A$.

Proof. Let $A=\{e\}^{\phi^{\prime}}$. By a theorem of Sacks which states that every recursively enumerable degree contains a regular recursively enumerable set 
([2, Theorem 1.22]), we may assume that $\emptyset^{\prime}$ is regular and recursively enumerable. Let $\left\{K_{r}\right\}_{r<\alpha}$ be a recursive list of all $\alpha$-finite sets. Then $A=\{e\}^{\phi^{\prime}}$ says that for each $\alpha$-finite set $K_{r}$.

$$
K_{r} \subset A \longleftrightarrow(\exists \nu, \eta)\left[(\gamma, 1, \nu, \eta) \in W_{e} \text { and } K_{r} \subset \emptyset^{\prime} \text { and } K_{r} \subset \alpha-\emptyset^{\prime}\right]
$$

and

$$
K_{\gamma} \subset \alpha-A \longleftrightarrow(\exists \nu, \eta)\left[(\gamma, 0, \nu, \eta) \in W_{e} \text { and } K_{r} \subset \emptyset^{\prime} \text { and } K_{r} \subset \alpha-\emptyset^{\prime}\right] .
$$

Fix $t<\alpha$. Since $A$ is regular by assumption, $A \mid t$ is $\alpha$-finite. Let this be $K_{r}$. Similarly the complement of $A$ restricted to $t$ is also $\alpha$-finite and equal to $a$ $K_{\delta}$. Thus there exist $\alpha$-finite sets $K_{\nu}, K_{\eta}, K_{\zeta}$, and $K_{\rho}$ such that

$$
(\gamma, 1, \nu, \eta) \in W_{e} \text { and } K_{\nu} \subset \emptyset^{\prime} \text { and } K_{\eta} \subset \alpha-\emptyset^{\prime}
$$

and

$$
(\delta, 0, \zeta, \rho) \in W_{e} \text { and } K_{\zeta} \subset \emptyset^{\prime} \text { and } K_{\rho} \subset \alpha-\emptyset^{\prime} \text {. }
$$

For a recursively enumerable set $C$, we let $C_{v}$ denote the set of elements enumerated in $C$ after $v$ steps of computation. By the regularity of $\emptyset^{\prime}$, there is a $v$ such that for all $v^{\prime} \geq v$,

$$
(\gamma, 1, \nu, \eta) \in W_{e, v} \text { and } K_{\nu} \subset \emptyset_{v^{\prime}}^{\prime} \text { and } K_{\eta} \subset \alpha-\emptyset_{v^{\prime}}^{\prime}
$$

and

$$
(\delta, 0, \zeta, p) \in W_{e, v^{\prime}} \text { and } K_{\zeta} \subset \emptyset_{v^{\prime}}^{\prime} \text { and } K_{\rho} \subset \alpha-\emptyset_{v^{\prime}}^{\prime}
$$

Let $R\left(x, v^{\prime}\right)$ express the relation

$(\exists \gamma, \delta)\left[K_{r}\right.$ and $K_{\delta}$ from a disjoint union of the ordinal $x+1$ and (1) holds].

Define

$$
p_{v}(x)= \begin{cases}1 & \text { if } x<v \text { and } R(x, v) \text { holds with } x \in K_{\tau} \\ 0 & \text { if } x<v \text { and } R(x, v) \text { holds with } x \in K_{\delta} .\end{cases}
$$

The $K_{r}$ and $K_{\delta}$ in the definition of $p_{v}(x)$ refer to the sets which make $R(x, v)$ true. We set $p_{v}(x)$, for $x \geq v$, to be 0 otherwise. It is now straightforward to verify that $\lim _{v \rightarrow \alpha} p_{v}=A$.

In general, if $B=\{e\}^{G}$, it is convenient to regard $\{e\}$ as being a partial recursive map from strings to strings, mapping $\alpha$-many initial segments of the characteristic function of $G$ into $\alpha$-many initial segments of the characteristic function of $B$. It is not difficult to see that $B$ is recursive in $G$ if and only if the initial segments of the characteristic function of $B$ may be computed using initial segments of the characteristic function of $G$. In what follows we shall assume that $\{e\}$ is a partial recursive map from strings to strings. 
Definition. Let $e$ be the index of a reduction procedure. Let a string $p$ be given. A string $p^{\prime}$ extending $p$ is said branch off $u$ times above $p$ if there is an $\alpha$-finite set $\left\{p_{v}\right\}, v<u$, of pairwise incompatible strings extending $p$ such that

(a) $p^{\prime} \cap p_{v}<p^{\prime} \cap p_{v^{\prime}}$ and $\{e\}^{p_{v}} \cap\{e\}^{p^{\prime}}<\{e\}^{p_{v^{\prime}}} \cap\{e\}^{p^{\prime}}$ for $v<v^{\prime}$;

(b) $\{e\}^{p_{v}}$ is incompatible with $\{e\}^{p_{v^{\prime}}}$ for $v \neq v^{\prime}$;

(c) $\{e\}^{p_{v}}$ is incompatible with $\{e\}^{p^{\prime}}$ for all $v<u$.

Lemma 2. Let $G$ be a 1-generic set and suppose that $B=\{e\}^{G}$ is not recursive. Then for all $p<G$, for all $u$, there exists an $r \geq p$ such that $r$ branches off at least $u$ times above $p$.

Proof. We prove this by induction on $u$. Let $p<G$. We will show that the string $r$ may be chosen to be an initial segment of $G$. Suppose that $u=s+1$ and that we have $r_{s}$ extending $p$ and an initial segment of $G$ such that $r_{s}$ branches off at least $s$ times above $p$ via the $\alpha$-finite sequence $\left\{p_{v, s}\right\}$, $v<s$. If all incompatible extensions of $r_{s}$ yield compatible strigs under the map $\{e\}$, in the sense that $\{e\}^{q}$ is compatible with $\{e\}^{r}$ for all $q, r$ extending $p$, then $B=\{e\}^{G}$ is necessarily recursive, since we will then immediately have an algorithm to compute $B$ : to decide if $x$ is in $B$, find a string $q$ extending $r_{s}$ such that $\{e\}^{q}$ has length greater than $x$, and compute the value of $\{e\}^{q}(x)$. This value must be $B(x)$. Since this is a contradiction, we know that there exist incompatible extensions of $r_{s}$ which yield incompatible strings under the map $\{e\}$. Furthermore, such pairs of incompatible strings exist above every initial segment of $G$ which extends $r_{s}$, else the same argument could be applied to prove the recursiveness of $B$. Now consider the collection $S$ of strings $q>r_{s}$ for which there is a string $r>r_{s}$ such that $\{e\}^{q},\{e\}^{r}$ are incompatible. By the above discussion and by the 1-genericity of $G$ one of the strings in $S$ is an initial segment of $G$. If we let this string be $r_{s+1}$, and the corresponding $q$ be $p_{s, s+1}$, then we have a string which is an initial segment of $G$, extends $p$, and branches off at least $s+1$ times above $p$ via the $\alpha$-finite sequence $\left\{p_{v, s+1}\right\}, v<s+1$, where $p_{v, s+1}=p_{v, s}$ for $v<s$.

We have shown that if $r_{s}$ extends $p$, is an initial segment of $G$, and branches off at least $s$ times above $p$, then there is an extension $r_{s+1}$ of $r_{s}$ which is an initial segment of $G$, such that $r_{s+1}$ branches off at least $s+1$ times above $p$, and that the $\alpha$-finite sequence $\left\{p_{v, s+1}\right\}, v<s+1$, extends the $\alpha$ finite sequence $\left\{p_{v, s}\right\}, v<s$.

Now let $u$ be a limit ordinal. Observe that given $r_{s}$ and $\left\{p_{v, s}\right\}, v<s$, one may obtain $r_{s+1}$ and $\left\{p_{v, s+1}\right\}, v<s+1$, using $G$ as an oracle. If we now assume that the same holds true for limit ordinals $u^{\prime}$ less than $u$, with the propərty that $\left\{p_{v, u^{\prime}}\right\}, v<u^{\prime}$, is the union of all those $\left\{p_{v, s}\right\}, v<s$ and $s<u^{\prime}$, then we may 
again take $r_{u}$ to be the union of $r_{s}, s<u$, and $\left\{p_{v, u}\right\}$ to be the union of all those $\left\{p_{v, s}\right\}, v<s$ and $s<u$. Then the maps $s \mapsto r_{s}$ and $s \mapsto\left\{p_{v, s}\right\}$ are weakly recursive in $G$, so that by the hyperregularity of $G$ (by the Proposition), both $r_{u}$ and $\left\{p_{v, u}\right\}, v<u$, are $\alpha$-finite. Notice now that $r_{u}$ extends $p$, is an initial segment of $G$, and branches off at least $u$ times above $p$. This proves Lemma 2.

A set is $\alpha$-G-finite if it is $A_{1}\left(L_{\alpha}, G\right)$ and bounded below $\alpha$. This turns out to be simply an element of $\left\langle L_{\alpha}, G\right\rangle$.

Definition. The $\Sigma_{1}\left(L_{\alpha}, G\right)$ projectum is the least $s$ for which there exists a subset of $s \Sigma_{1}$ definable in $\left\langle L_{\alpha}, G\right\rangle$ which is not $\alpha$-G-finite.

Recall that sets $\Sigma_{1}$ definable in $\left\langle L_{a}, G\right\rangle$ are precisely those which are r.e. in $G$. Let $G$ be 1-generic. By [2, Corollary 1.31] and by the Proposition, a function is $\Sigma_{1}$ definable in $\left\langle L_{\alpha}, G\right\rangle$ if and only if it is weakly recursive in $G$, and by [2, Proposition 1.33] a function is $\Delta_{1}$ in $\left\langle L_{\alpha}, G\right\rangle$ if and only if it is recursive in $G$. Again by our earlier Proposition, elements of $\left\langle L_{\alpha}, G\right\rangle$ are simply elements of $L_{\alpha}$, and are therefore $\alpha$-finite. Thus we may replace the phrase 'an element of $\left\langle L_{\alpha}, G\right\rangle$ ' in the definition above by 'an element of $L_{\alpha}$ ', i.e. $\alpha$-finite.

Lemma 3. Let $G$ be a 1-generic set. Then there is an injective function f recursive in $G$ mapping $\alpha$ into the $\Sigma_{1}$ projectum of $\left\langle L_{\alpha}, G\right\rangle{ }^{*}$

Proof. Let $A$ be a subset of the $\Sigma_{1}$ projectum of $\left\langle L_{\alpha}, G\right\rangle$ which is $\Sigma_{1}$ definable in $\left\langle L_{\alpha}, G\right\rangle$ but not $\alpha$-finite. By the regularity and hyperregularity of $G$, there is a one-one function $f$ partial recursive in $G$ which enumerates $A$. The function $f$ has domain $\alpha$ since otherwise the enumeration of $A$ ends at some stage $s$, and that would imply that $A$ is $\alpha$-finite, contradicting our choice of $A$.

Lemma 4. Let $G$ be a 1-generic set recursive in $\emptyset^{\prime}$. Let $u_{0}$ be the $\Sigma_{1}$ projectum of $\left\langle L_{\alpha}, G\right\rangle$. Then there is a recursive surjection $g: \alpha \times u_{0} \rightarrow \alpha$ such that for all $u_{1}<u_{0}$, there is an $s_{1}$ such that

$$
g(s, u)=g\left(s_{1}, u\right) \text { for all } s>s_{1} \text { and all } u<u_{1},
$$

i.e. $g$ converges uniformly on all $u_{1}<u_{0}$.

Proof. By Lemma 1, let $\left\{p_{s}\right\}$ be a recursive sequence of strings converging uniformly to $G$. Since $G$ is 1 -generic, there are $\alpha$-infinitely many among these strings which are initial segments of $G$. Let $f$ be a function

*Strictly speaking, this should be denoted $\left\langle L_{\alpha}[G], \varepsilon, G\right\rangle$. 
recursive in $G$ mapping $\alpha$ injectively into $u_{0}$ given by Lemma 3 . Let $\{d\}^{G}$ be the characteristic function of the graph of $f$ (again we do not distinguish between $G$ and its characteristic function in this case). Let $\left\{q_{s}\right\}$ be the set of strings which are images of strings in $\left\{p_{s}\right\}$ under $\{d\}$. Then we have $f=$ $\lim _{s \rightarrow \alpha} q_{s}$, i.e. for all $t$ there is an $s_{0}$ such that graph $(f)\left|t=q_{s}\right| t$ for all $s>s_{0}$. This means that for the pair $(x, u)<t, f(x)=u$ if and only if $q_{s}((x, u))=1$ for all $s>s_{0}$. Observe also that for each $u_{1}<u_{0}$, there is a sufficiently large $s$, denoted by $s_{1}$, such that no $s>s_{1}$ satisfies $q_{s}((x, u))=1$ for any $x$ and for any $u<u_{1}$ not in the image of $f$. To see this, notice firstly that by the choice of $u_{0}$ and $u_{1}<u_{0}$, the set $K$ of ordinals $u<u_{1}$ not in the image of $f$ is $\alpha$-finite. Now if every initial segment of $G$ is extended by a string $p$ such that $\{d\}^{p}$ maps $(x, u)$ to 1 for some $u$ in $K$ and for some $x$, then by the 1 -genericity of $G$ this happens to $G$ as well, namely there is an initial segment $p^{\prime}$ of $G$ such that $\{d\}^{p^{\prime}}$ maps $(x, u)$ to 1 for some $u$ in $K$ and for some $x$. But this means that $f(x)=u$, contradicting our choice of $u$. Thus there is an initial segment $r$ of $G$ for which no extension $p=p_{s}$ satisfies $\{d\}^{p^{\prime}}((x, u))=1$ for some $x$ and for any $u$ in $K$. This in turn implies that there is an $s_{1}$, such that for all $s>s_{1}, q_{s}((x, u)) \neq 1$ for any $u$ in $K$ and for any $x$. We now define $g$ by

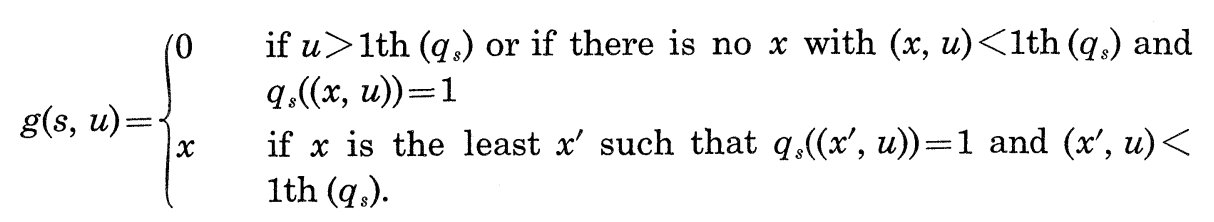

We verify that the function $g$ satisfies the requirement. Let $u_{1}<u_{0}$ be given. Then the image set of $f$ restricted to $u_{1}$ is $\Sigma_{1}$ definable in $\left\langle L_{\alpha}, G\right\rangle$ and so $\alpha$-finite, by the choice of $u_{0}$. It follows that $f^{-1}$ with domain restricted to $u_{1}$ has image bounded in $\alpha$ by some $w$ since $f^{-1}$ is recursive in $G$ and $G$ is hyperregular. Let $t$ be chosen such that for $x<w$ and $u<u_{1}$, the pair $(x, u)$ is less than $t$. Then there is an $s_{0}$ such that for $x<w$ and $u<u_{1}$ (so that $(x, u$ ) $<t), f(x)=u$ if and only if $q_{s}((x, u))=1$ for all $s>s_{0}$. Assume also that $s_{0}$ has been chosen so that no $s>s_{0}$ satisfies $q_{s}((x, u))=1$ for any $x$ and any $u<u_{1}$ not in the image of $f$. By the definition of $g$, this means that for all $s>s_{0}$, $g(s, u)=g\left(s_{1}, u\right)$ for all $u<u_{1}$. This proves Lemma 4 .

By abuse of notation, we write $g(u)=\lim _{s \rightarrow \alpha} g(s, u)$ in the above lemma. Note that $g$ is one-one on the set of ordinals $u$ such that $g(u) \neq 0$.

Let $B$ be a non-recursive set recursive in a 1-generic set $G$ which is recursive in $\emptyset^{\prime}$. We now construct a 1 -generic set $C$ recursive in $B$. The function $g$ will be the 'priority function'. Let $K_{d}$ be the $d t h \alpha$-finite collection of r.e. sets of strings, defined uniformly over all $d$. Then each $K_{d}=K_{g(u)}$ for some $u$. Since the preimage of 0 under $g$ is not uniquely defined (indeed 
the set of preimages has order type $\alpha$ ), we will only consider $\left\{K_{d} \mid d \geq 1\right\}$. Since in any effective enumeration of the $\alpha$-finite sets, there always exist two sets which are identical but having different indices (as easily verified by the Recursion Theorem), there is no loss of generality in considering only indices starting with 1.

Let $\{e\}^{G}=B$. Let $\left\{p_{v}\right\}$ be a recursive set of strings converging to $G$. We define a recursive function $k$ from strings to strings such that

(1) $k$ is total on $B$;

(2) let $S(q, d)$ express the relation

every recursively enumerable set of strings in $K_{d}$ contains an element extended by $q$.

Fix $d$. If for all $t$ there is a $q>k(B) \mid t$ such that $S(q, d)$ holds, then the set $\left\{v \mid(\exists r)\left[r>p_{v}\right.\right.$ and $q=k\left(\{e\}^{r}\right)$ and $\left.\left.S(q, d)\right]\right\}$ has $\alpha$ many elements.

Let $C=k\left(\{e\}^{a}\right)$. We show that the set $C$ is 1 -generic. Suppose that $K_{d}$ satisfies the hypothesis of (2). Then by the conclusion of (2) every initial segment of $G$ is extended by some string $p$ which is mapped under $e$ and then $k$ onto a string which extends an element of each r.e. set in $K_{d}$. The set of such strings $p$ is r.e. By the 1-genericity of $G$, one of these extensions, say $p^{*}$, is an initial segment of $G$. Since $\{e\}^{p^{*}}$ is an initial segment of $B$, we see that $k\left(\{e\}^{p^{*}}\right)$ is an initial segment of $C$.

On the other hand, suppose that for all strings $q$ extending $C \mid t$, it is not the case that $q$ extends an element of every r.e. set in $K_{d}$. If every r.e. set in $K_{d}$ contains an element extended by some initial segment of $C$ which extends $C \mid t$, then one can define a map taking the index of each r.e. set $R$ of strings in $K_{d}$ to the length of the least initial segment of $C$ extending $C \mid t$ which extends an element of $R$. This map is recursive in $G$ (since $C$ is recursive in $G$ ) with an $\alpha$-finite domain. By the hyperregularity of $G$ its image is bounded below $\alpha$. Then the union of these initial segments of $C$ is again an initial segment $q$ of $C$, but having the property that every r.e. set in $K_{d}$ contains an element which is extended by $q$. This again contradicts the choice of $C \mid t$. Thus there is an r.e. set in $K_{d}$, say $R$, such that no initial segment of $C$ extending $C \mid t$ extends an element of $R$. For the singleton $\{R\}$, by the previous paragraph it must then be the case that there is an initial segment $q^{\prime}$ of $C$ extending $C \mid t$ such that no extension of $q^{\prime}$ extends an element of $R$. It then follows that for all extensions $q^{*}$ of $q^{\prime}$, no element of $R$, now considered to be in $K_{d}$, is extended by $q^{*}$. This implies that $C$ is 1 -generic.

We construct $k$ by induction. At stage $s$, first find the least string $p_{v}$ (in the $\left.\left\{p_{v}\right\}_{v<\alpha}\right)$ having an extension which branches off at least $s$ times above $p_{v}$, such that none of these strings (the extension and the branchings) is defined under $k \circ e$ thus far (we often write, for convenience, $\{e\}^{p}$ as $e(p)$ for a string $p)$. By Lemma 2 such a string $p_{v}$ always existes. Let the least such $p_{v}$ be 
$p_{v(s)}$ and let its least extension which branches off at least $s$ times over $p_{v(s)}$ be $p_{v(s)}^{\prime}$. Let $p_{v(s)}^{\prime \prime}$ be the largest substring of $p_{v(s)}$ defined under $k \circ e$. Observe that the notion of the 'largest substring' is well-defined since we define $k$ such that $k \circ e$ is closed under limits (i.e. if $p=\cup p_{v}$ and $k\left(e\left(p_{v}\right)\right)$ is defined for all $v$, then $\left.k(e(p))=\cup k\left(e\left(p_{v}\right)\right)\right)$.

We continue to set $u_{0}$ be the $\Sigma_{1}$ projectum of $\left\langle L_{\alpha}, G\right\rangle$. Compute $s$ steps to find the least $u<u_{0}$, called $u(s)$, such that $g(s, u) \neq 0$ and there is a string $q$ of length at most $s$ above $k\left(e\left(p_{v(s)}^{\prime \prime}\right)\right)$, having the property that every r.e. set of strings in $K_{g(s, u)}$ contains an element extended by $q$. Furthermore $q$ is not in the image of $k \circ e$ by step $s$. Let the least such $q$ be chosen and denoted $q^{*}$. Let $s^{\prime}$ be the length of $q^{*}$ above $k\left(e\left(p_{v(s)}^{\prime \prime}\right)\right)$. Let $p_{v(s)}^{*}$ be the unique substring of $p_{v(s)}^{\prime}$ which branches off exactly $s^{\prime}$ times above $p_{v(s)}$. Set $k\left(e\left(p_{v(s)}^{*}\right)\right)$ $=q^{*}$. For $s^{\prime \prime}<s$, let $p_{s^{\prime \prime}}^{*}$ be the $s^{\prime \prime}$ th string (in the order of the lengths of the intersection with $p_{v(s)}^{*}$ ) extending $p_{v(s)}^{\prime \prime}$ in the collection of strings witnessing the fact that $p_{v(s)}^{*}$ branches off exactly $s^{\prime}$ times above $p_{v(s)}^{\prime \prime}$. There are two cases to consider:

Case 1. The ordinal $s^{\prime \prime}$ is a limit ordinal. Then we do not define $k\left(e\left(p_{s^{\prime \prime}}^{*}\right)\right)$.

Case 2. The ordinal $s^{\prime \prime}$ is of the form $t+1$. Let $k\left(e\left(p_{s^{\prime \prime}}^{*}\right)\right)$ be the string of least length which extends $q^{*} \mid 1$ th $\left(k\left(e\left(p_{v(s)}^{\prime \prime}\right)\right)\right)+t$ and incompatible with $q^{*}$. The picture to be kept in mind for this case is that once $q^{*}$ is defined under $k \circ e$, all the strings whose lengths are successor ordinals less than 1 th $\left(q^{*}\right)$, which extend $k\left(e\left(p_{v(s)}^{\prime \prime}\right)\right)$, and are incompatible with $q^{*}$ only at the greatest ordinal in the domain, are included in the image of $k \circ e$ (see figure).

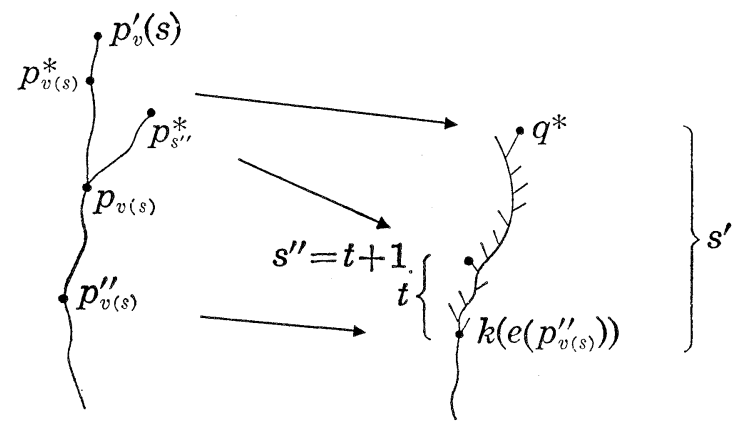

This completes the definition of the recursive map $k$ at stage $s$. Since the sequence $\left\{p_{v}\right\}$ converges to $G$, we see that $k \circ e$ is total on $G$, and hence $k$ is total on $e(G)$. We now verify that $k(e(G))$ is 1-generic by induction on $K_{g(u)}, u<u_{0}$.

Fix $u_{1}<u_{0}$. Suppose that for each $u<u_{1}$, there is an initial segment $q^{u}$ of $C=k(e(G))$ in the image of $k \circ e$ under $G$ such that either every r.e. set in $K_{g(u)}$ contains an element extended by $q^{u}$, or there is an r.e. set in $K_{g(u)}$ 
which contains no element extending $q^{u}$.

ClaIm 1. $\cup q^{u}, u<u_{1}$, is $\alpha$-finite.

Let $s_{1}$ be a stage such that, by Lemma $4, g(u)=g(s, u)=g\left(s_{1}, u\right)$ for all $u<u_{1}$. Let $k\left(e\left(p_{v(s(u))}^{*}\right)\right)=q_{u}$. Then the set $K$ of $u$ 's less than $u_{1}$ such that $k\left(e\left(p_{v(s(u))}^{*}\right)\right)$ extends an element of every r.e. set in $K_{g(u)}$ (remember that $g(u)$ $\left.=g\left(s_{1}, u\right)\right)$ is r.e. in $G$. By the definition of the ordinal $u_{0}, K$ is $\alpha$-finite. Then the map taking each $u$ in $K$ to the length of $k\left(e\left(p_{v(s(u))}^{*}\right)\right)=q^{u}$ is recursive in $G$, and by the hyperregularity of $G$ must be bounded below $\alpha$. Claim 1 follows immediately as a consequence of this.

ClaIm 2. If every initial segment of $C$ is extended by a string which extends an element of evəry r.e. set in $K=K_{g\left(u_{1}\right)}$, then every initial segment of $C$ is extended by one such string which is in the image of $k \circ e$.

Suppose that $K$ satisfies the hypothesis. For all stages $s>s_{1}$, we have $g\left(s, u_{1}\right)=g\left(s_{1}, u_{1}\right)=g\left(u_{1}\right)$. Let us also assume without loss of generality that at all stages $s$ after $s_{1}$, all strings extending $p_{v}, v>s_{1}$, are mapped onto strings which extend $q=\cup q^{u}$ given in Claim 1 . This assumption is permissible since the sequence $\left\{p_{v}\right\}$ converges to $G$ and $C=k(e(G))$. From now on we only consider stages $s>s_{1}$. Let $R$ be the r.e. set of strings which extend an element of each r.e. set in $K$. Consider $C \mid t$. For each $r$ in $R$ extending $C \mid t$, let $r^{\prime \prime}$ be its largest substring defined under $k \circ e$ by the stage $s$ where $r$ is enumerated in $R$. By the way the function $k$ is defined, we know that the lengths of the $r^{\prime \prime}$ s tend to $\alpha$. Let $R^{\prime \prime}$ be the set of such $r^{\prime \prime}$ s. Then every initial segment of $G$ is extended by some string $p^{\prime \prime}$ which is mapped onto some string in $R^{\prime \prime}$. It follows by the 1 -genericity of $G$ that there is one (hence order type $\alpha$ many) $p^{\prime \prime}$ which is an initial segment of $G$, and so there is one (hence order type $\alpha$ many) $r^{\prime \prime}$ which is an initial segment of $C$. Notice that if by a stage $s$ where an $r$ in $R$ is enumerated there is a proper extension of $r^{\prime \prime}$, say $r^{*}$, defined under $k \circ e$, then by the construction (see Case 2 where $k\left(e\left(p_{s^{\prime}}^{*}\right)\right)$ is defined for all successor ordinal $\left.s^{\prime \prime}\right)$ we must have $r \mid 1$ th $\left(r^{\prime \prime}\right)+1$ in the image of $k \circ e$ as well, contradicting the choice of $r^{\prime \prime}$ as the largest substring of $r$ defined under $k \circ e$ by stage $s$. It follows that by stage $s$, there is no proper extension of $r^{\prime \prime}$ defined under $k \circ e$. Now by the above there are order type $\alpha$ many initial segments of $C$ each of which assumes the form $r^{\prime \prime}$ of a string $r$ in $R$ at stage $s$ (for order type $\alpha$ many $s$ ), hence order type $\alpha$ many $r^{\prime \prime}$ 's will at later stage be extended by strings in the image of $k \circ e$. Since these happen at stages after $s$, by our construction the strings $r$ in $R$ which extend these $r^{\prime \prime}$ 's will be chosen to be the string $q^{*}$ (see the definition of $q^{*}$ in the construction above) at the appropriate stage, since $K_{g\left(u_{1}\right)}$ is accorded the highest priority at any stage after $s_{1}$. We have shown that every initial 
segment of $C$ is extended by a string $r$ in $R$ which is in the image of $k \circ e$, hence assumes the form $q^{*}$. By (2), this implies that the set $C$ is 1-generic.

The proof of the main theorem is complete. This will be applied in a paper under preparation to show that for strongly $\Sigma_{2}$ inadmissible constructible cardinals (such as $\omega_{\omega}^{L}$ ), there is no 1-generic degree bounding a minimal degree below $\emptyset^{\prime}$ ('see the forthcoming paper: 1-generic degrees and minimal degrees in higher recursion theory, II'). From our point of view, this seems to indicate that for such admissible ordinals, minimal degrees below $\emptyset^{\prime}$ do not exist.

\section{Appendix.}

We prove in this appendix that the apparently simpler definition of 1genericity given in this paper is equivalent to the one given in [2]. The only difference in the two definitions appears in the case where $\alpha^{*}$, the least ordinal which contains an r.e. non- $\alpha$-finite set, is a singular cardinal of $L_{\alpha}$. In this case, a set $G$ as defined in [2] is 1-generic if and only if for all $\alpha$-finite collections $K$ of r.e. sets such that the $\alpha$-cardinality of $K$ is less than $\alpha^{*}$, there is an initial segment $p$ of $G$ such that either $p$ extends an element of every r.e. set in $K$ or there is an r.e. set in $K$ for which no element is extended by any extension of $p$. We will show that the condition 'the $\alpha$ cardinality of $K$ is less than $\alpha^{*}$ may be removed. This will imply that the two definitions are actually equivalent.

Let $G$ be 1-generic in so far as $\alpha$-finite collections $K$ of $\alpha$-cardinality less than $\alpha^{*}$ is concerned. To differentiate this from the definition of 1-genericity given in this paper, let us label it simply as $1^{\prime}$-genericity. Let $K^{\prime}$ be an $\alpha$ finite collection of r.e. sets of strings. We will obtain an initial segment $p$ of $G$ such that either $p$ extends an element of every r.e. set in $K$, or there is an r.e. set in $K$ not containing any extension of $p$. A basic fact in $\alpha$-recursion theory says that since $\alpha^{*}<\alpha$, it is the largest cardinal in $L_{\alpha}$. Thus we may assume that the $\alpha$-cardinality of $K$ is $\alpha^{*}$ (otherwise the conclusion is immediate). Let the members of $K$ be listed as $\left\{S_{e} \mid e<\alpha^{*}\right\}$. Let $\kappa$ be the cofinality of $\alpha^{*}$. Thus there is an $\alpha$-finite increasing function $h$ mapping $\kappa$ cofinally into $\alpha^{*}$. Let $K(u)=\left\{S_{e} \mid e<h(u)\right\}$. Then we get a strictly increasing sequence of collections of r.e. sets, with each $K(u)$ having cardinality less than $\alpha^{*}$ that the definition of $1^{\prime}$-genericity applies to each member of the sequence. Suppose that for some $u$, there is an initial segment $p$ of $G$ and a member of $K(u)$ no element of which extends any extension of $p$. Then this string $p$ fulfills our definition of 1 -genericity with regard to the $\alpha$-finite collection $K$.

On the other hand, suppose that for all $u<\kappa$, there is an initial segment 
$p^{u}$ of $G$ such that every member of $K(u)$ contains an element extended by $p^{u}$. Then one obtains a function mapping $u$ to the length of $p^{u}$. This function is recursive in $G$, and by the hyperregularity of $G$ [2, Lemma 2.5], the function is actually $\alpha$-finite since its domain is $\kappa$. Then the union of $p^{u}, u<\kappa$, is an initial segment of $G$ which extends an element of each r.e. set in $K$. Thus $1^{\prime}$-genericity implies 1-genericity. The other direction is immediate. We conclude that these two definitions are equivalent.

\section{REFERENCES}

[1] C. T. Chong, Recursion theory on strongly $\Sigma_{2}$ inadmissible ordinals, Proc. Oberwolfach 1984 Logic Conference, Springer Verlag, 1985.

[2] C. T. Chong, Techniques of Admissible Recursion Theory, Springer Verlag, 1984.

[ 3 ] C. T. Chong, Minimal degrees recursive in 1-generic degrees, (submitted).

[4] C. T. Chong and C. G. Jockusch, Minimal degrees and 1-generic degrees below $\emptyset^{\prime}$, Proc. Aachen Logic Conference, Springer Verlag, 1984.

[5] R. M. Friedberg, A criterion for completeness of degrees of unsolvability, J. Symbolic Logic, 22 (1957), 159-160.

[ 6 ] S. D. Friedman, Negative solutions to Post's problem, II., Ann. of Math., 113 (1981), 25-43.

[ 7 ] C. G. Jockusch, Degrees of generic sets, Proc. Leeds Logic Conference, Cambridge Univ. Press, 1981.

[ 8 ] C. G. Jockusch and D. B. Posner, Double jumps of minimal degrees, J. Symbolic Logic, 43 (1978), 715-724.

[ 9 ] D. B. Posner and R. W. Robinson, Degrees joining to J. Symbolic Logic, 46 (1981), 714-722.

[10] S. G. Simpson, Degree theory on admissible ordinals, Generalized Recursion Theory, North Holland, 1974.

NATIONAL UNIVERSITY OF SINGAPORE 